\title{
Clinical and Pathological Changes in the Offspring of Rats with Torsio Uteri
}

\author{
Risvanli $\mathrm{A}^{*_{1}}$, Timurkaan $\mathrm{N}^{2}$, Saat $\mathrm{N}^{3}$, Dogan $\mathrm{H}^{1}$ and Seker $\mathrm{I}^{4}$ \\ ${ }^{1}$ Department of Obstetrics and Gynecology, Faculty of Veterinary Medicine, University of Firat, Elazig, Turkey \\ ${ }^{2}$ Department of Pathology, Faculty of Veterinary Medicine, University of Firat, Elazig, Turkey \\ ${ }^{3}$ Department of Obstetrics and Gynecology, Faculty of Veterinary Medicine, University of Balikesir, Balikesir, Turkey \\ ${ }^{4}$ Department of Zootechny, Faculty of Veterinary Medicine, University of Firat, Elazig, Turkey
}

${ }^{*}$ Corresponding author: Risvanli A, Department of Obstetrics and Gynecology, Faculty of Veterinary Medicine, University of Firat, Elazig, Turkey 23159, Fax: 0090424238 8173, Tel: 0090424237 0000, E-mail: arisvanli@firat.edu.tr

Citation: Risvanli A, Timurkaan N, Saat N, Dogan H, Seker I (2017) Clinical and Pathological Changes in the Offspring of Rats with Torsio Uteri. J Vet Sci Ani Husb 5(3): 302

Received Date: June 08, 2017 Accepted Date: October 23, 2017 Published Date: October 25, 2017

\begin{abstract}
We investigated the histopathological changes observed in the brains, organs, and apoptotic changes in the brain of young rats born from rats with torsio uteri that survive. We divided 10 Sprague-Dawley rats with a gestational age of 18-19 days into two groups. The rats in group 1 (i.e., the control group; $n=5$ ) were given anesthesia only; the rats in group 2 (study group; $n=5$ ) underwent a 360 -degree clockwise torsion in their uterus, and the torsion was corrected after 6 hours. The births of the offspring were followed, and 7 female and male offspring 1 month of age were selected in each group. The vaginal openings of the young female offspring were checked to determine the time to reach puberty. The 1-month-old male offspring and the mature young female rats were then decapitated. Tissue samples, including visceral organs, genital organs, and brains, were evaluated by histapathologically; we also investigated apoptosis via the TUNEL method. Although no abortion was observed in any of the pregnant rats in the control group, two abortions were observed in the study group after torsion/detorsion. The survival rate difference between the groups was statistically significant. The time to reach puberty was longer in female rats born from rats with torsio uteri; however, this difference was not statistically significant. Microscopic lesions were not observed in the visceral organs, genital organs, or brain. In addition, apoptosis were not determined in the brains of the experimental or control-group rats.
\end{abstract}

Keywords: Torsio uteri; Offspring; Rats

\section{Introduction}

Torsio uteri-the twisting of the uterus along its long axis-are observed commonly in ruminants, particularly in cattle. Torsio uteri are one of the causes of dystocia in cows, and it has been reported in occur in $7 \%$ of all cases of dystocia. The onset of torsio uteri may occur at any time during pregnancy starting at the second month. However, it is evident at the time of birth and particularly during the first stage of birth or within the last 2 months prior to birth [1,2]. The prognosis is good if torsio uteri is noted sufficiently early. Depending on the incidence of hypoxia or a prolonged birth time, calf mortality in $24-71 \%$ of cases has been reported [3]. The fetus within the uterus may be exposed to hypoxia or anoxia due to stenosis in the umbilical cord during torsion. Damage may be observed during detorsion as well. Ischemia/reperfusion damage has been observed as a result of torsion and detorsion of the uterus [4-6].

It has been suggested that mammalian fetuses and newborns are more resistant to anoxia or hypoxia compared with adults of the same species because anaerobic metabolism is activated in the case of anoxia of fetuses or newborns. As a result, less energy is needed [5,7]. Again, blood circulation is primarily used for important organs such as the brain, heart, and suprarenal glands in fetuses and newborns in the case of anoxia or hypoxia. However, permanent brain damage has been demonstrated in dogs, rhesus monkeys, and guinea pigs in cases of prolonged anoxia. Other disorders have also been shown to be related to the damage [5,7].

There have been only a few studies of experimentally induced torsio uteri in laboratory animals during pregnancy. However, Erlwanger et al. [6] reported $270^{\circ}$ torsion in the right cornu of a pregnant Sprague-Dawley rat.

Many studies have been reported on the effects of fetal hypoxia on offspring [5,7]. It is well known that cows are exposed to fetal hypoxia, especially with the cause of torsio uteri, and fertility is low in healthy offspring [5,7]. However, there is little information 
about the pathogenesis of this infertility. This study was carried out to determine the effects on puberty age and possible pathological changes in brain, genital organs, and visceral organs of live born male and female offspring of fetal hypoxia experimentally induced by torsio-uteri in rats.

\section{Materials and Methods}

The study was designed in two steps: in vivo and in vitro.

\section{In vivo Procedures}

Animals: A total of 10 Sprague-Dawley female rats aged 3-4 months and a gestational age of 18-19 days weighing between 200 and 250 grams were divided into two groups. We assembled a control group $(n=5)$ and a study group $(n=5)$. We used a total of 28 infant rats, 14 from each group ( 7 females and 7 males each). The animals were obtained from The Laboratory Animals Investigation Center of Firat University (Elazig, Turkey). During the study, the rats were housed in individual cages in a 12-hour light/12-hour dark cycle. They were fed food and water ad libitum. Ethical committee approval was obtained from the local ethics committee of the Firat University Laboratory Animals Department (24.02.2015 - 2016/22).

Design of the groups: The animals were grouped as follows:

Group 1 (control): Pregnant rats with a gestational age of 18-19 days underwent anaesthesia only $(n=5)$.

Group 2 (study): Six hours of 360-degree torsion was created in the uterus of pregnant rats with a gestational age of 18-19 days $(\mathrm{n}=5)$.

Vaginal Irrigations and Determination of the Day of Pregnancy: Vaginal irrigations were performed as described by Sahna and Risvanli [8]. The irrigations were performed using distilled water, an elastic pipette, and pipette tips. The fluid obtained after the irrigation was pipetted onto a slide and examined under the microscope with $40 \times$ magnification. The densities of the cell types observed in the slides prepared were evaluated as,+++ , and +++ . The rats with a superficial cellular density of +++ were accepted as being in their oestrus period. The animals with spermatozoid detected in the slides prepared using their vaginal irrigations were accepted as having had coitus. That date was recorded as the 0th day of pregnancy.

Operations: Rompun (Bayer HealthCare LLC, Animal Health, Berlin) (10 mg/kg IM) - Ketalar (Pfizer Limited, Kent) (90 mg/kg IM) combination anesthesia was administered to the rats during the operations [9]. A laparotomy was performed under general anesthesia following the routine procedures. The right cornu uteri of the animals were then passed through the hole formed in the non-vascular region of the lig. lata uteri of the left cornu uteri at the level of the vesica urinaria. Next, the right cornu was passed through the same hole for the second time, and clockwise torsion of $360 \mathrm{o}$ was created in both cornu uteri. Following this procedure, the abdomen of the animals was closed using the proper suturing material. Six hours after the operation, the same animals underwent a laparotomy again under general anesthesia, and the torsion was corrected. The abdomens were then closed again.

The births of the rats ensued, and 7 female and 7 male infants that were 1 month old were selected from each group to be included in the study.

The vaginal openings of the female young rats were checked to determine the time at which they attained puberty. The male rats and the female rats that had reached puberty were then decapitated. All of the animals were anesthetized with ether prior to decapitation.

\section{In vitro Procedures}

\section{Immunohistochemistry and Histopathological Tests}

For the histopathological examination, we collected tissue samples of the liver, kidneys, heart, lungs, spleen, ovaries, testes, and brain. The tissue samples were fixed in $10 \%$ neutral buffered formalin, routinely processed, embedded in paraffin, sectioned at 5 $\mathrm{mm}$ thickness, and stained with hematoxylin and eosin for histologic examination. In addition, we analyzed the brain sections using the terminal deoxynucleotidyl transferase dUTP nick end-labeling (TUNEL) method to detect apoptosis. The TUNEL staining was performed using an Apoptag Plus Kit (Merck Millipore, USA) according to the manufacturer's recommended protocol. Briefly, after deparaffinized the brain sections with xylene and graded concentrations of alcohol, they were stored in a citrate buffer solution at $\mathrm{pH} 6.0$ in a microwave oven for $15 \mathrm{~min}$ (distilled water was added every $5 \mathrm{~min}$ ) for antigen retrieval. Endogenous peroxidase activity was quenched with $3 \%$ hydrogen peroxide. The sections were then incubated with TdT enzyme in a humidified chamber at $37^{\circ} \mathrm{C}$ for $1 \mathrm{~h}$. After incubation with anti-digoxigenin-peroxidase for $30 \mathrm{~min}$ at room temperature, peroxidase was detected using diaminobenzidine [10]. The specimens were then washed with distilled water and counterstained with Mayer's hematoxylin. Formalin-fixed, paraffin-embedded mouse testis tissue belonging to our previous study and known to be TUNEL positive in our laboratory was used as positive control tissue in the TUNEL staining.

\section{Statistical Analysis}

Among the non-parametric tests, we used the Mann-Whitney $U$ test to compare two independent groups and analyze the data from the rats' vaginal openings. Among the chi-squared tests, we used Fisher's exact test to compare the abortion data between the groups, and we used the Pearson chi-squared test to compare the survival powers of the infants. 


\section{Results}

\section{Metagenomic Sequencing}

Although no abortions were observed in any of the pregnant rats in the control group, two abortions were observed in the study group after torsion/detorsion. No statistical significance was determined between the groups with regard to abortion rates $(P>0.05)$ (Table 1).

The survival rates were $76.1 \%$ for the control group and $51.9 \%$ for the study group, and this difference was statistically significant $(P<0.05)$ (Table 1$)$.

\begin{tabular}{|c|c|c|c|c|c|c|c|c|}
\hline & \multicolumn{4}{|c|}{ Abortus } & \multicolumn{4}{|c|}{ Survival rate of offspring } \\
\hline & \multicolumn{2}{|l|}{+} & \multicolumn{2}{|l|}{-} & \multicolumn{2}{|c|}{ Living } & \multicolumn{2}{|c|}{ Dead } \\
\hline & Frequency & $\%$ & Frequency & $\%$ & Frequency & $\%$ & Frequency & $\%$ \\
\hline $\begin{array}{c}\text { Group } 1 \text { (Control) } \\
(n=5)\end{array}$ & 0 & 0.0 & 5 & 100.0 & 35 & 76.1 & 11 & 23.9 \\
\hline $\begin{array}{c}\text { Group } 2 \text { (Study) } \\
(n=5)\end{array}$ & 2 & 40.0 & 3 & 60.0 & 14 & 51.9 & 13 & 48.1 \\
\hline $\mathbf{P}$ & \multicolumn{4}{|c|}{0.444} & \multicolumn{4}{|c|}{0.033} \\
\hline
\end{tabular}

Table 1: Abortion rates in the study group and survival rates of the infants born from these mothers

We observed at the end of the study that two female infant rats born from mothers with torsio uteri died before reaching puberty. Data on the vaginal openings are summarized in Table 2. Our statistical analyses revealed no difference between the groups $(P>0.05)$. There were no significant microscopic lesions in the brain, heart (Figure 1A,B,C and D), liver, kidney, lung, spleen, uteri, testes or ovaries - of any rats (Figure $1 \mathrm{~A}$ and B). The organs investigated in the study revealed a normal histological structure. While positivity were observed in the positive control rat tissue (Figure 1E), TUNEL positivity was not detected in the brains of either the experimental or control-group rats (Figure $1 \mathrm{~F}$ ).

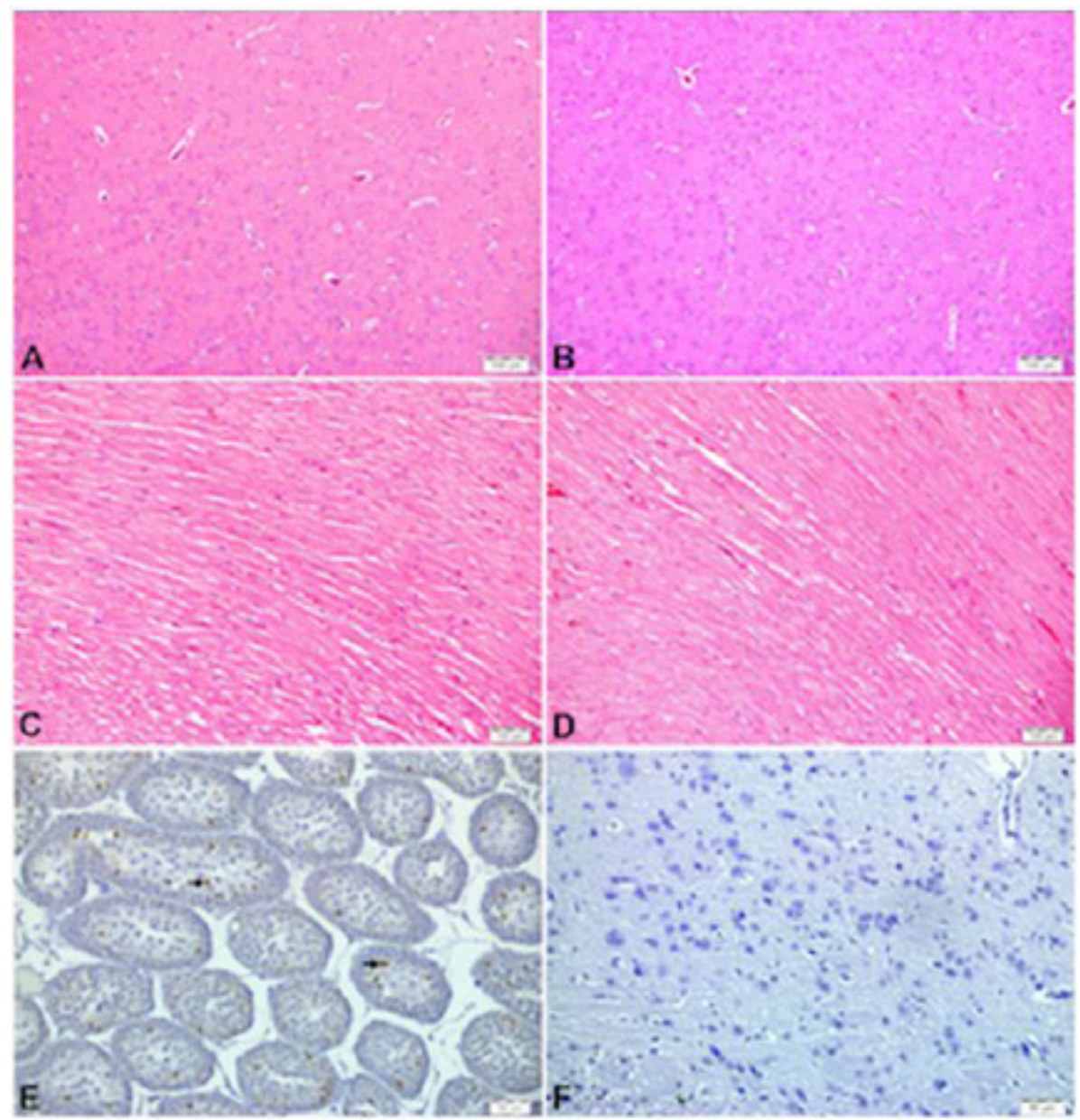

Figure 1: Microscopic pictures of the study (A and B) Photomicrographs showing normal brain histology in a male rat of control (A) and experimental group (B); Haematoxylin and Eosin (C and D); Photomicrographs showing normal heart histology in a female rat of control (C) and experimental group (D); Haematoxylin and Eosin (E); Photomicrograph of the positive control mouse testis tissue. Note that apoptotic cells (arrows) by TUNEL staining. Mayer's hematoxylin counterstain; (F) Photomicrograph of the brain of a male rat in experimental group showing no positivity by TUNEL staining. Mayer's hematoxylin counterstain 


\begin{tabular}{|c|c|c|}
\hline & $\begin{array}{c}\text { Group 1 } \\
(\text { Day) } \\
(\mathbf{n}=7)\end{array}$ & $\begin{array}{c}\text { Group 2 } \\
(\text { Day }) \\
(\mathbf{n}=5)\end{array}$ \\
\hline Vaginal Opening & $54.86 \pm 1.16$ & $60.80 \pm 6.71$ \\
\hline
\end{tabular}

The difference between values was not statistically significant $(P<0.05)$

Table 2: Days of vaginal openings of the infants

\section{Discussion}

Uterine torsion is a pregnancy pathology commonly observed in ruminants, particularly cows. A literature search revealed no studies pertaining to the effects of anoxia/hypoxia on rats born from rats with torsio uteri. Although a fertility reduction has been reported in live births from rats with torsio uteri, its pathogenesis has not yet been clearly defined. However, it is expected that anoxia/hypoxia, which occurs at the moment of uterine torsion, affects the genital organs as well as other organs of the fetus. To determine whether this hypothesis is correct, the age at which the offspring born from uterine torsion mothers reached puberty were followed up, but there was no difference between the groups. However, in order to determine the effect of uterine torsion on the fertility of the offspring, the reproductive endocrinology and gamete biology of such offspring should be studied.

Generally, resistance against anoxia or survival regresses with pregnancy progression. The brain functions of fetuses decrease in order to protect infants from anoxia that ensues as a result of ruptures, cuts, or twists in the umbilical cord.

In an animal model, hypoxic and ischemic damage results from an operation termed "retarded Cesarean section." In this operation, the umbilical cords of the fetuses are clamped at a time close to birth, and blood circulation and thereby oxygen circulation are blocked [11,12]. Another method used for this purpose is ligation of the $a$. uterine media alone or in combination with $a$. ovarica [13]. In these models, hypoxia developed due to chronic hypoxia and chronic placental hypoperfusion. In these kinds of models, systemic hypoxia or anoxia was observed instead of a targeted organ.

Hypoxia threatens brain functions at every stage of life starting with early fetal development to old age. Hypoxia generally impairs intracellular $\mathrm{Ca}^{+2}$ homeostasis and leads to various pathological situations such as neuronal cell damage, neurodegeneration, and cell death. The damage that occurs varies as a function of the duration of hypoxia as a result of ischemia according to fetal age [14-16]. We demonstrated that the survivals rates of infant rats born to rats with torsio/detorsio uteri were decreased. However, there were no differences between the surviving infants and the control group in terms of histopathological findings observed in the brain, visceral, or genital organs of the infant rats and TUNEL-positive animals. We accordingly concluded that the torsion procedure performed on pregnant rats had a negative effect on the survival rates of infant rats. These findings suggest that the torsio-uterine may not have affected all of the fetuses at the same level. In addition, it can be said that the torsio-uterine was not significantly important in puberty age in the offspring but prolonged.

\section{Conclusion}

A literature search revealed no studies of laboratory animals experimentally undergoing torsio/detorsio uteri and the hypoxia or anoxia of the infants born from these animals. We observed that infant rats born from rats that had experimentally undergone torsio uteri at advanced stages of their pregnancy and survived were not affected by light microscopic level at the puberty.

\section{Acknowledgement}

This study was supported by the TUBITAK - $115 \mathrm{O} 381$.

\section{References}

1. Jackson PG (2004) Handbook of Veterinary Obstetrics (2 ${ }^{\text {nd }}$ Edn) Elsevier, Philadelphia, United States.

2. Lyons NA, Gordon PJ, Borsberry S, Macfarlane J, Mouncey J (2013) Clinical forum: Bovine uterine torsion: a review. Livestock 18: 18-24.

3. Noakes DE, Parkinson TJ, England GCW (2009) Veterinary Reproduction and Obstetrics (9th Edn) Saunders, Elsevier, Philadelphia, United States.

4. Lyons NA, Knight JTJD, Aldridge BM, Gordon PJ (2013) Incidence, management and outcomes of uterine torsion in dairy cows. Cattle Pract 21: 1-6.

5. Jochems CEA, van der Valk JB, Stafleu FR, Baumans V (2002) The use of fetal bovine serum: ethical or scientific problem? Altern Lab Anim 30: $219-27$.

6. Erlwanger KH, Costello MA, Meyer LC (2011) Uterine torsion in a Sprague Dawley rat (Rattus norvegicus). J S Afr Vet Ass 82: 183-4.

7. Jensen A (1996) The brain of the asphyxiated fetus - basic research. Eur J Obstet Gynecol Reprod Biol 65: 19-24.

8. Sahna KC, Risvanli A (2015) Th1/Th2 cytokine balance and SOCS3 levels of female offspring born from rats with gestational diabetes mellitus. Kafkas Univ Vet Fak Derg 21: 837-40.

9. Risvanli A, Aydin M, Kaygusuzoglu E, Timurkan H (2003) The effect of thyroidectomy on sexual cycle and pregnancy rates in rats. Turkish J Vet Anim Sci 27: 873-7.

10. Oberhause SM (2003) TUNEL and idouble labeling assay for apoptotic cell with specific antigens. Meth Mol Biol 218: 85-96.

11. Bjelke B, Andersson K, Ogren SO, Bolme P (1991) Asphyxic lesion: proliferation of tyrosine hydroxylase-immuno-reactive nerve cell bodies in the rat substantia nigra and functional changes in dopamine neurotransmission. Brain Res 543: 1-9.

12. Shen Y, Isaacson RL, Smotherman WP (1991) The behavioural and anatomical effects of prenatal umbilical cord clamping in the rat and their alteration by the prior maternal administration of nimodipine. Restor Neut Neurosci 3: 11-22. 
13. Chanez C, Rabin O, Heroux M, Giguere JF (1993) Cerebral amino acid changes in an animal model of intrauterine growth retardation. Metab Brain Dis 8: 61-72. 14. Halici Z, Karaca M, Keles ON, Borekci B, Odabasoglu F, et al. (2008) Protective effects of amlodipine on ischemia-reperfusion injury of rat ovary: biochemical and histopathologic evaluation. Fert Steril 90: 2408-15.

15. Nyakas C, Buwalda B, Luiten PG (1996) Hypoxia and brain development. Prog Neurobiol 49: 1-51.

16. Karaca M, Odabasoglu F, Kumtepe Y, Albayrak A, Cadirci E, et al. (2009) Protective effects of erythropoietin on ischemia/reperfusion injury of rat ovary. Eur J Obstet Gynecol Reprod Biol 144: 157-62.

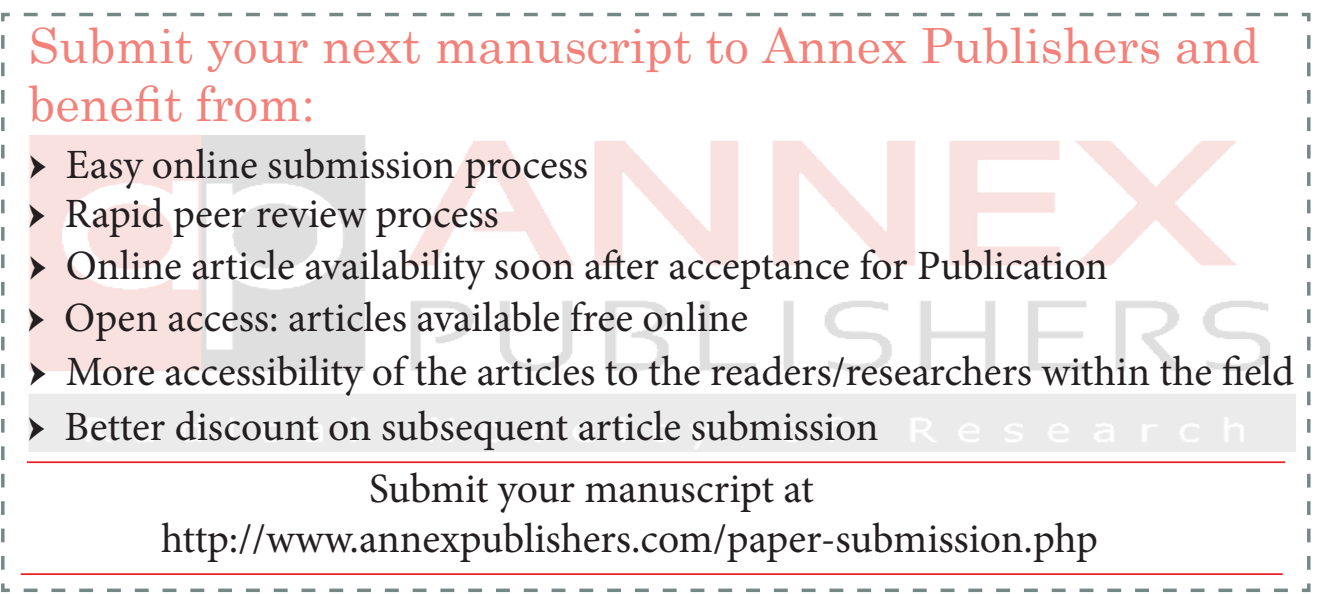

